\title{
Extranodal presentation of a lymphoma with precursor B-cell phenotype and translocation $t(8 ; 14)$ in South Africa
}

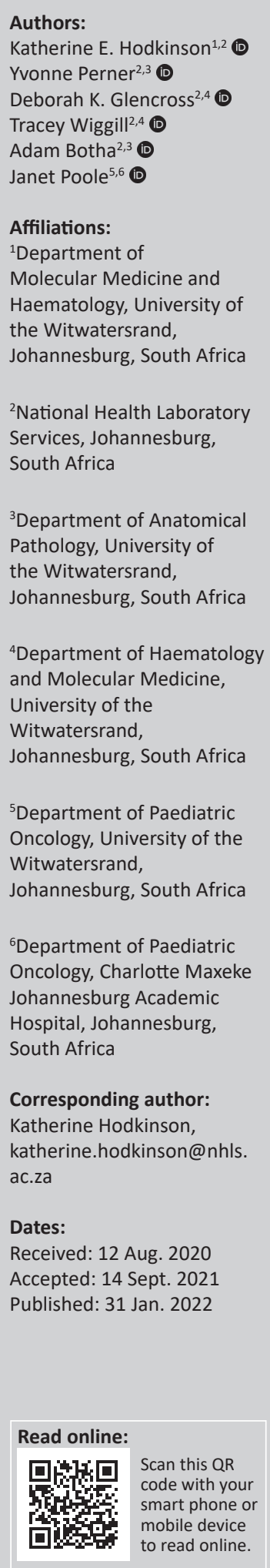

Introduction: A rare entity of a B-cell malignancy with precursor B-cell phenotype and concomitant translocation $\mathrm{t}(8 ; 14)$ or variant MYC translocation exists. These cases show clinical, pathological and molecular overlap between precursor B-lymphoblastic leukaemia or lymphoma and Burkitt leukaemia or lymphoma (BLL).

Case presentation: We report a case from February 2019 at the Charlotte Maxeke Johannesburg Academic Hospital, South Africa, of a 9-month-old infant with a predominantly extracranial soft tissue mass showing extradural extension. There was no involvement of the peripheral blood or bone marrow. Fine needle aspiration and Tru-Cut biopsy of the soft tissue scalp mass showed the tumour to be of precursor B-cell phenotype. Contrastingly, an immunophenotypic assessment revealed a high S-phase fraction and raised concern for BLL. This prompted testing for the translocation $\mathrm{t}(8 ; 14)$ by fluorescence in-situ hybridisation analysis, which confirmed this aberration.

Management and outcome: Based on the published experience of other centres, the patient was initiated on a BLL protocol. Despite an excellent clinical response, the patient succumbed to neutropenic sepsis six months after diagnosis.

Conclusion: Leukaemia or lymphoma with translocation $\mathrm{t}(8 ; 14)$ or variant MYC translocation and precursor B-cell phenotype is a rare entity with a varied clinical presentation. This poses a challenge for diagnosis and classification and a clinical dilemma for the choice of treatment.

Keywords: Burkitt leukaemia/lymphoma; B-lymphoblastic leukaemia/lymphoma; translocation $\mathrm{t}(8 ; 14)$; variant MYC translocations; S-phase fraction; terminal deoxynucleotidyl transferase (TdT); extranodal.

\section{Introduction}

The presence of the translocation $\mathrm{t}(8 ; 14)$ or variant MYC translocation in leukaemia with a precursor B-cell phenotype has been described in approximately $2 \%$ of paediatric cases. ${ }^{1,2}$ Rarer still is a pure lymphomatous version (extranodal or nodal), which, to the best of our knowledge, has only been reported twice in the literature, one of which was in a paediatric patient. ${ }^{3,4}$

This entity has overlapping clinical and pathological features with Burkitt leukaemia or lymphoma (BLL) and precursor B-lymphoblastic leukaemia or lymphoma (B-ALL). It has been described across a spectrum of ages ranging from 32 months to 64 years, in both male and female patients. Peripheral blood and bone marrow involvement have been reported in the majority of cases, with isolated extranodal disease reported in only a few. ${ }^{1,3,4,5}$ The immunophenotype is that of a precursor B-cell, with the expression of CD19, CD10 and terminal deoxynucleotidyl transferase (TdT), varied expression of CD34, and no expression of both surface light chain and CD20. Molecular studies have confirmed the involvement of the MYC gene in a translocation $t(8 ; 14)$ or variant translocation, which is the molecular hallmark of BLL. ${ }^{2}$ This genetic aberration has largely directed the choice of therapy towards a mature B-cell or Burkitt-like protocol in almost all reported cases. It is uncertain whether this represents the best therapeutic approach as there is very limited data on both the duration of remission in these patients and the exact underlying molecular behaviour of this tumour.

This case study highlights this rare entity, as well as its associated diagnostic and therapeutic challenges.

How to cite this article: Hodkinson KE, Perner Y, Glencross DK, Wiggill T, Botha A, Poole J. Extranodal presentation of a lymphoma with precursor B-cell phenotype and translocation t(8;14) in South Africa. Afr J Lab Med. 2022;11(1), a1355. https://doi.org/10.4102/ajlm. v11i1.1355

Copyright: @ 2022. The Authors. Licensee: AOSIS. This work is licensed under the Creative Commons Attribution License. 


\section{Ethical considerations}

Parental informed consent was provided for this case report. The ethical clearance was obtained from the University of the Witwatersrand Human Research Ethics Committee (Medical), Johannesburg, South Africa, under the approval number M190356. Patient results were de-identified and stored in a secure database to ensure patient confidentiality.

\section{Case presentation}

A 9-month-old male patient from Angola presented to the Charlotte Maxeke Johannesburg Academic Hospital, South Africa, in February 2019, with a 3-4-week history of progressive bilateral proptosis and scalp masses. The computed tomography scan identified bilateral occipital and temporal scalp masses with extracranial extension into the orbital cavities and sinuses, and extradural extension into the anterior cranial fossa. There was no hepatosplenomegaly or lymphadenopathy. A fine needle aspiration sample of the soft tissue scalp mass was submitted for flow cytometry, and a Tru-Cut biopsy (manufacturer unknown) for histological assessment at the National Health Laboratory Service. As part of the staging work up, bone marrow aspiration and a trephine biopsy were performed to exclude infiltration of the marrow.

\section{Laboratory methods}

Flow cytometry was performed on the fine needle aspiration sample from the scalp mass on a FACS Calibur instrument (BD Biosciences, San Jose, California, United States), using the PAINT-A-GATE (Becton Dickinson, BD Biosciences, San Jose, California, United States) and MODFIT (Verity Software House, Topsham, Maine, United States) software. The following antibodies were used: CD19 FITC (Becton Dickinson, BD Biosciences, San Jose, California, United States), CD10 PE (Beckman Coulter Inc., Brea, California, United States), CD45 PERCP (Becton Dickinson, BD Biosciences, San Jose, California, United States), CD34 APC (Becton Dickinson, BD Biosciences, San Jose, California, United States), CD117 PE (Beckman Coulter Inc., Brea, California, United States), HLA-DR FITC (Becton Dickinson, BD Biosciences, San Jose, California, United States), CD13 PE (Beckman Coulter Inc., Brea, California, United States), Kappa FITC (Dako, Glostrup, Denmark), Lambda PE (Dako, Glostrup, Denmark) and cytoplasmic TdT FITC (Dako, Glostrup, Denmark).

Immunohistochemical work up was performed on the TruCut biopsy from the scalp mass using the following stains: CD20 (Dako, Glostrup, Denmark), PAX5 (Dako, Glostrup, Denmark), CD10 (Leica Biosystems, Newcastle Upon Tyne, United Kingdom), TdT (Cell Marque, Rocklin, California, United States), CD34 (Dako, Glostrup, Denmark), EBER ISH (Roche, Mannheim, Germany), BCL2 (Dako, Glostrup, Denmark), and Ki67 (Dako, Glostrup, Denmark).
Fluorescence in-situ hybridisation for the detection of translocation $\mathrm{t}(8 ; 14)$ was performed using a Vysis LSI MYC/ IGH/CEP8 tri-colour dual fusion probe (Abbott, Chicago, Illinois, United States), with orange, green and aqua signals reflecting the $\mathrm{MYC}, \mathrm{IgH}$ and centromere 8 regions.

\section{Laboratory results}

Histological examination of the Tru-Cut biopsy of the scalp mass revealed intermediate-sized tumour cells with irregular nuclear contours, a high nuclear-cytoplasmic ratio, dispersed nuclear chromatin, and one to four inconspicuous nucleoli present within a prominent background of tingible body macrophages (Figure 1). The tumour immunophenotype based on flow cytometry and immunohistochemistry was that of a precursor B-cell, with the expression of TdT, absence of surface light chains and a high Ki67 index (Table 1, Table 2 and Figure 2). Contrastingly, the high S-phase fraction detected was in a range that is typically seen in Burkitt leukaemia. ${ }^{6}$ Given the latter, Fluorescence in-situ hybridisation analysis was requested and found to be positive for the translocation $\mathrm{t}(8 ; 14)(\mathrm{q} 24 ; \mathrm{q} 32)$ (Figure 3).

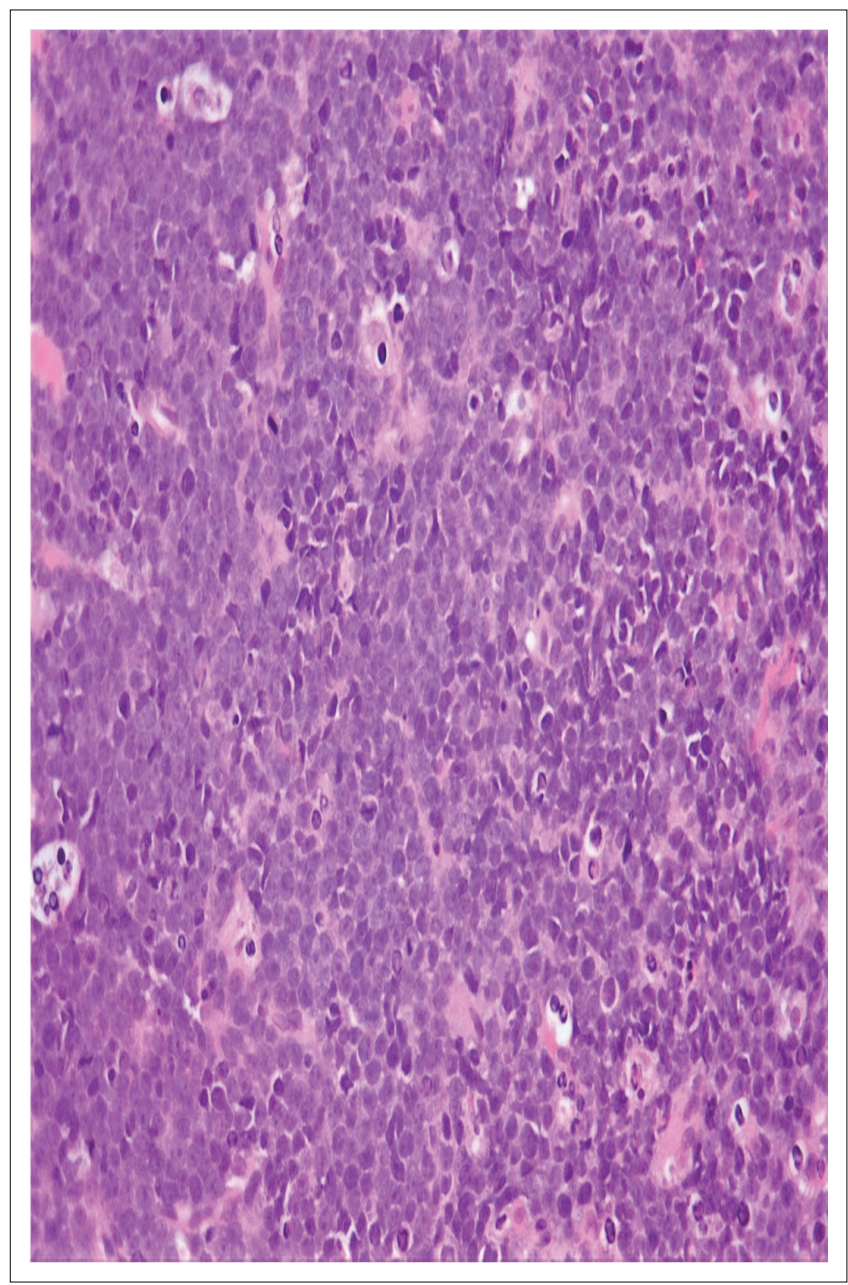

FIGURE 1: Tru-Cut biopsy of scalp mass of a 9-month-old male patient who presented to the Charlotte Maxeke Johannesburg Academic Hospital in February 2019. Image shows diffuse infiltration by intermediate-sized tumour cells with dispersed nuclear chromatin, irregular nuclear contours and one to four inconspicuous nucleoli. Numerous tingible body macrophages impart a starry sky pattern. 7 mitotic figures per 40 HPF. Haematoxylin \& eosin (H\&E) stain at $\times 40$ magnification. 
TABLE 1: Laboratory results at presentation of a 9-month-old male patient who presented to the Charlotte Maxeke Johannesburg Academic Hospital in February 2019.

\begin{tabular}{lc}
\hline Laboratory tests & Results \\
\hline Blood results & \\
White cell count $\left(\times 10^{9} / \mathrm{L}\right)$ & 5.8 \\
Absolute neutrophil count $\left(\times 10^{9} / \mathrm{L}\right)$ & 2.95 \\
Haemoglobin (g/dL) & 7.5 \\
Mean cell volume (f/L) & 65.4 \\
Mean cell haemoglobin concentration (g/dL) & 29 \\
Platelet count ( $\left.\times 10^{9} / \mathrm{L}\right)$ & 734 \\
LDH (U/L) & 682 \\
Ferritin (ug/L) & 13 \\
Uric acid (mmol/L) & 0.22 \\
Bone marrow infiltration (Based on morphology, IgH & Absent \\
gene rearrangement studies, cytogenetic analysis) & \\
Cerebrospinal fluid involvement (based on cytology, & Absent \\
flow cytometry, IgH gene rearrangement studies) & \\
\hline
\end{tabular}

IgH, Immunoglobulin heavy chain; LDH, Lactate dehydrogenase.

TABLE 2: Laboratory results at presentation of a 9-month-old male patient who presented to the Charlotte Maxeke Johannesburg Academic Hospital in February 2019.

\begin{tabular}{|c|c|c|}
\hline Immunophenotype & FNA Flow cytometry & Tru-Cut biopsy IHC \\
\hline CD19 & $\begin{array}{c}+ \\
\text { (bright) }\end{array}$ & NT \\
\hline CD20 & NT & - \\
\hline CD22 & NT & NT \\
\hline PAX5 & NT & + \\
\hline CD10 & $\begin{array}{c}+ \\
\text { (bright) }\end{array}$ & + \\
\hline $\begin{array}{l}\text { Human leukocyte antigen - DR } \\
\text { isotype }\end{array}$ & + & NT \\
\hline CD45 & $\begin{array}{l}+ \\
\text { (dimmer than the } \\
\text { background lymphocytes) }\end{array}$ & NT \\
\hline TdT & + in $\sim 30 \%$ & + in $\sim 30 \%-40 \%$ \\
\hline CD34 & - & - \\
\hline CD117 & - & NT \\
\hline CD13 & $\operatorname{dim}+$ & NT \\
\hline Light chain restriction & - & NT \\
\hline S-phase fraction & $35.7 \%$ & NT \\
\hline Ki67 & NT & $\sim 100 \%$ \\
\hline BCL2 & NT & + \\
\hline EBER ISH & NT & - \\
\hline FISH analysis $\mathrm{t}(8 ; 14)(\mathrm{q} 24 ; \mathrm{q} 32)$ & + & + \\
\hline IgH gene rearrangement studies & Monoclonal & NT \\
\hline
\end{tabular}

negative; +, positive; BCL2, B-cell lymphoma 2; EBER ISH, Epstein-Barr virus-encoded small RNA in-situ hybridisation; FISH, fluorescence in-situ hybridisation; FNA, fine needle aspirate IgH, Immunoglobulin heavy chain; IHC, immunohistochemistry on the Tru-Cut biopsy of the scalp mass; LDH, Lactate dehydrogenase; NT, not tested; TdT, Terminal deoxynucleotidyl transferase.

\section{Management and outcomes}

The patient was initiated on a BLL regimen as per the FAB LMB 95 protocol with excellent clinical response. ${ }^{7}$ There was complete resolution of the proptosis and the scalp masses were no longer clinically evident. Sadly, the patient succumbed to neutropenic sepsis six months after diagnosis.

\section{Discussion}

The co-existence of the translocation $t(8 ; 14)$ or variant MYC translocation with a precursor B-cell phenotype in an extranodal malignancy is extremely uncommon and has been reported in only one paediatric and one adult patient in the

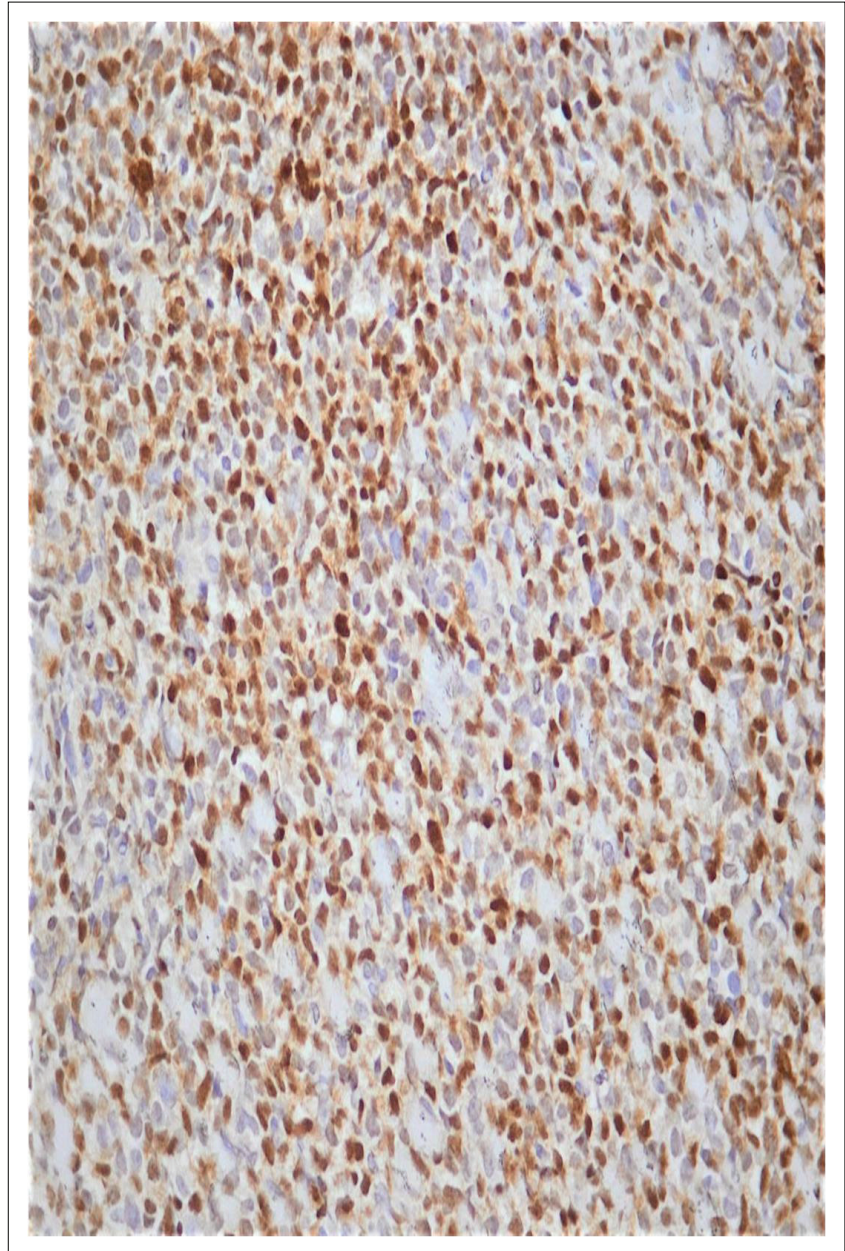

FIGURE 2: Terminal deoxynucleotidyl transferase (TdT) immunohistochemical stain of Tru-Cut biopsy of scalp mass from a 9-month-old male patient who presented to the Charlotte Maxeke Johannesburg Academic Hospital in February 2019. Image shows $\sim 40 \%$ positivity in the tumour. $\times 40$ magnification.

literature. ${ }^{3,4}$ These individuals were both female and had no laboratory evidence of either peripheral blood or bone marrow involvement at presentation. The youngest, a 5-year-old, presented with abdominal, orbital and mandibular masses. ${ }^{4}$ The second, a 64-year-old, had multiple extranodal lesions and a chronic disease course. ${ }^{3}$ Similarly, the clinical presentation of our patient was with isolated extranodal disease. This pattern of involvement is often seen in Burkitt lymphoma and thus formed part of the differential diagnosis in our patient.

Laboratory work up demonstrated the co-expression of CD19 and CD10 by the tumour, with no expression of CD34 or light chains. Because of this, the expression of TdT was assessed. Terminal deoxynucleotidyl transferase is a DNA polymerase that functions to provide junctional diversity in both B-cell and T-cell receptor genes at the precursor cell stage. ${ }^{8}$ It is a marker of immaturity and its expression in mature neoplasms such as BLL would be aberrant. Terminal deoxynucleotidyl transferase was found to be positive in $\sim 30 \%-40 \%$ of the tumour on both the fine needle aspiration and Tru-Cut biopsy specimens. While these results pointed towards a tumour at a precursor cell stage of development, the S-phase fraction analysis found the tumour proliferative activity to be very high. This measurement is performed by flow cytometry 


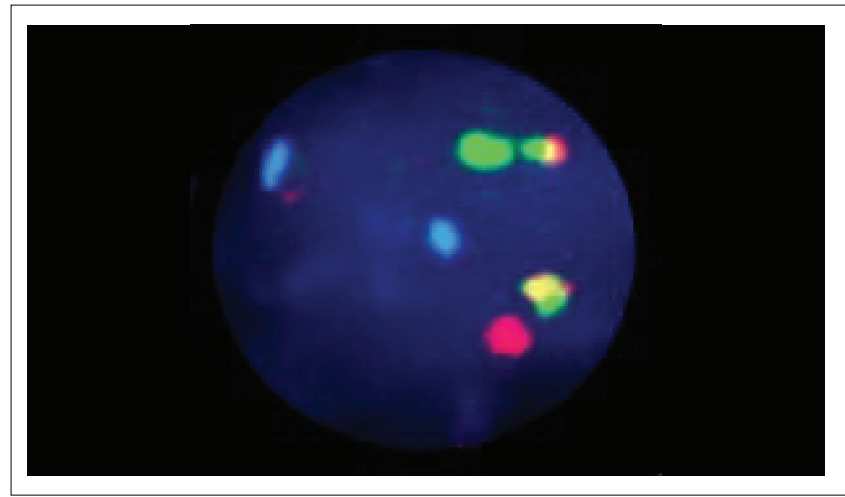

Source: Image courtesy of the Somatic Cell Genetics Unit (Department of Molecular Medicine and Haematology, National Health Laboratory Service, Johannesburg), February 2019

FIGURE 3: Fluorescence in-situ hybridisation analysis of fine needle aspirate from a 9-month-old male patient who presented to the Charlotte Maxeke Johannesburg Academic Hospital in February 2019. Image shows translocation t(8;14)(q24;q32) using the Vysis LSI MYC/IGH/CEP8 tri-colour dual fusion probe, as well as a 2F1O1G2A pattern: 2 fusion, 1 orange, 1 green, 2 aqua signals. The orange, green and aqua signals represent the MYC, IgH and centromere 8 regions.

and uses DNA content to determine the proportion of cells in each phase of the cell cycle. Studies performed at our centre have shown the S-phase fraction of B-ALL to be characteristically around 10, whereas a fraction of more than 30 , as seen in our patient, would be more typical of BLL. 6 Comparisons could not be made with the other cases in the literature as S-phase fraction analysis was not reported. The Ki67 index detects a protein associated with cellular proliferation, which is present in all active stages of the cell cycle. Given that the Ki67 index and S-phase analysis measure different aspects of proliferation, these parameters are not always comparable. Notably, the Ki67 index is unhelpful in distinguishing B-ALL and BLL as values of over 95\% can be anticipated in both. ${ }^{6}$ Despite the conflicting laboratory findings in our patient, the high S-phase fraction in the context of extranodal disease prompted the testing for, and confirmation of, the translocation $\mathrm{t}(8 ; 14)$. B-cell lymphoma 2 (BCL2) expression is typically seen in acute lymphoblastic leukaemia and the possibility of a double-hit lymphoma was excluded by the presence of TdT. ${ }^{2}$ S-phase fraction analysis may therefore serve as an early indicator of this disease entity, albeit further investigation of its clinical utility is required. In summary, a combination of diagnostic modalities with evaluation by a multidisciplinary team is required to confirm this rare entity. We propose a laboratory approach to the diagnosis of lymphomas with features of B-ALL and BLL (Figure 4).

The therapeutic management of BLL differs significantly from B-ALL. The former requires high-intensity pulsed chemotherapy that is tailored to the high proliferative rate of the tumour. Furthermore, the duration of therapy is shorter as compared to the extended maintenance used in B-ALL. ${ }^{7}$ Our patient was instituted on a BLL treatment protocol, in line with the therapeutic approach described in the literature. ${ }^{1,4,5}$ There are however limited cases and minimal long-term follow-up data from which meaningful conclusions can be drawn. On a molecular level, there is a lack of consensus as to whether this entity represents a precursor B-cell at an intermediate stage of

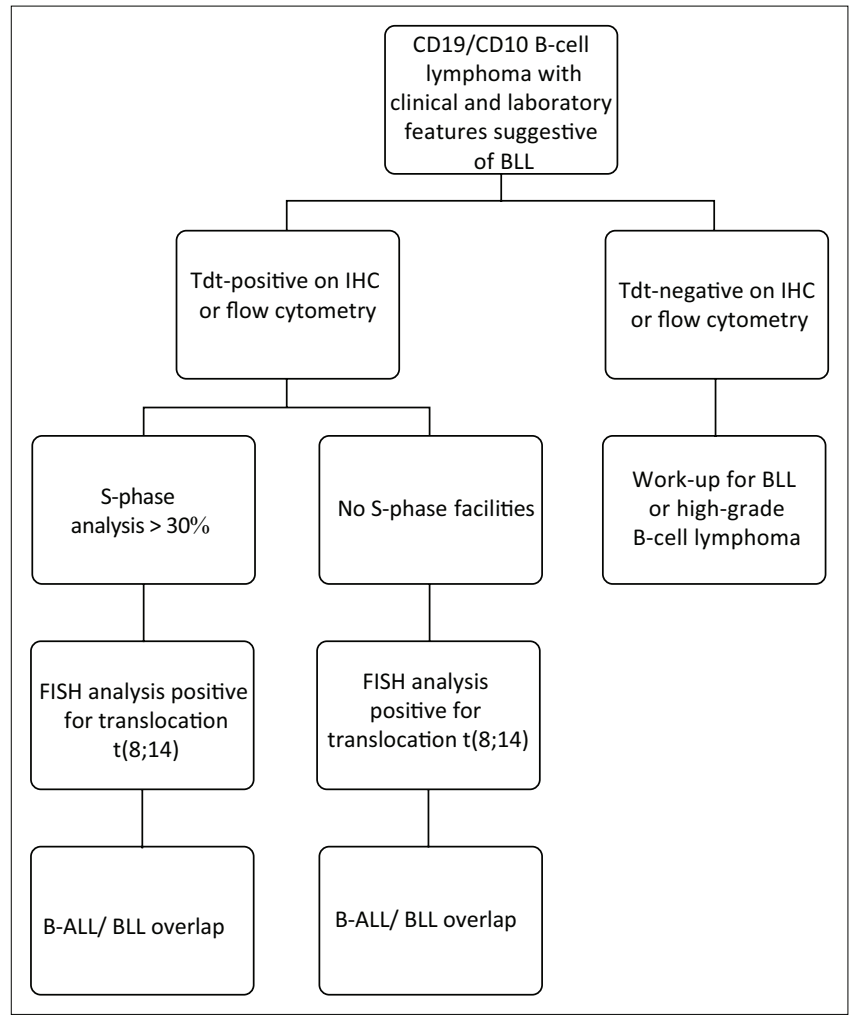

BLL, Burkitt leukaemia or lymphoma; B-ALL, precursor B-lymphoblastic leukaemia or lymphoma; TdT, terminal deoxynucleotidyl transferase; IHC, immunohistochemistry; FISH, fluorescence in-situ hybridisation.

FIGURE 4: Laboratory approach to the diagnosis of lymphoma with features of precursor B-lymphoblastic leukaemia or lymphoma and Burkitt leukaemia or lymphoma.

maturation and with aberrant MYC expression, or a mature B-cell with a less differentiated immunophenotype.

Furthermore, questions have been raised as to whether or not the presence of the translocation $t(8 ; 14)$ translates into an actual proliferation advantage. In an attempt to address these questions, one study analysed the molecular characteristics of neoplasms positive for IG-MYC rearrangement and with precursor B-cell phenotype in 12 patients. Aberrant variable diversity joining recombination was shown in five patients and activating $\mathrm{N}$ - and K-RAS mutations were detected in seven; all neoplasms had a DNA methylation profile that clustered with that of precursor B-cells. In light of these findings, the authors of the study posed the question as to whether or not cases with MYC rearrangement better fit a B-ALL profile. ${ }^{9}$ What is apparent from the literature is that further studies are required into the molecular behaviour of this entity to determine the prognostic implications and best therapy.

\section{Conclusion}

Leukaemia or lymphoma with translocation $t(8 ; 14)$ or variant MYC translocation and precursor B-cell phenotype is a rare entity with varied clinical presentation. Awareness of this entity and a high index of suspicion are required by both clinicians (haematologists, oncologists) and pathologists to prevent a misdiagnosis. Further studies into the molecular and clinical behaviour of this tumour are required to optimise the therapeutic approach. 


\section{Acknowledgements}

Thanking both Ashleigh Forsman and Tshilidzi Dzivhani from the Somatic Cell Genetics Unit (Department of Molecular Medicine and Haematology, National Health Laboratory Service, Johannesburg) for the analysis of the fluorescence in-situ hybridisation.

\section{Competing interests}

The authors declare that they have no financial or personal relationships that may have inappropriately influenced them in writing this article.

\section{Authors' contributions}

K.E.H. drafted the manuscript. All authors were involved in the conceptualisation, structuring and editing of the final manuscript.

\section{Sources of support}

This research received no specific grant from any funding agency in the public, commercial or not-for-profit sectors.

\section{Data availability}

Data sharing is not applicable to this article as no new data were created or analysed in this study.

\section{Disclaimer}

The views and opinions expressed in this article are those of the authors and do not necessarily reflect the official policy or position of any affiliated agency of the author.

\section{References}

1. Navid F, Mosijczuk AD, Head DR, et al. Acute lymphoblastic leukemia with the $(8 ; 14)(q 24 ; q 32)$ translocation and FAB L3 morphology associated with a B-precursor immunophenotype: The Pediatric Oncology Group experience. Leukemia. 1999;13(1):135-141. https://doi.org/10.1038/sj.leu.2401244

2. Swerdlow SH, Campo E, Pileri SA, et al. The 2016 revision of the World Health Organization classification of lymphoid neoplasms. Blood. 2016;127(20): 2375-2390. https://doi.org/10.1182/blood-2016-01-643569

3. Shiratori S, Kondo T, Fujisawa S, et al. C-MYC rearrangement in B-cell lymphoblastic lymphoma with the involvement of multiple extranodal lesions. Leuk Lymphoma. 2011;52(4):716-718. https://doi.org/10.3109/10428194.2010 .551158

4. Meznarich J, Miles R, Paxton CN, Afify Z. Pediatric B-Cell lymphoma with lymphoblastic morphology, TdT expression, MYC rearrangement, and features overlapping with Burkitt lymphoma. Pediatr Blood Cancer. 2016;63(5):938-940. https://doi.org/10.1002/pbc.25907

5. Sakaguchi K, Imamura T, Ishimaru S, et al. Nationwide study of pediatric B-cell precursor acute lymphoblastic leukemia with chromosome 8q24/MYC rearrangement in Japan. Pediatric Blood Cancer. 2020;67(7):e28341. https://doi. org/10.1002/pbc.28341

6. Glencross DK. Flow cytometry in diagnostic haematopathology. Johannesburg: University of the Witwatersrand; 1992.

7. Patte C, Auperin A, Gerrard M, et al. Results of the randomized international FAB/LMB96 trial for intermediate risk B-cell non-Hodgkin lymphoma in children and adolescents: It is possible to reduce treatment for the early responding patients. Blood. 2007;109(7):2773-2780. https://doi.org/10.1182/ blood-2006-07-036673

8. McCaffrey R, Harrison TA, Parkman R, Baltimore D. Terminal deoxynucleotidy transferase activity in human leukemic cells and in normal human thymocytes. N Engl J Med. 1975;292(15):775-780. https://doi.org/10.1056/ NEJM197504102921504

9. Wagener R, López C, Kleinheinz K, et al. IG-MYC (+) neoplasms with precursor B-cell phenotype are molecularly distinct from Burkitt lymphomas. Blood. 2018;132(21):2280-2285. https://doi.org/10.1182/blood-2018-03-842088 